\title{
The Review on Eye Tracking Technology Application in Digital Learning Environments
}

\author{
Aleksandrs GORBUNOVS \\ Distance Education Study Centre, Faculty of E-Learning Technologies and Humanities, Riga \\ Technical University, Kronvalda Blvd.1, Riga, LV-1010, Latvia \\ aleksandrs.gorbunovs_1@rtu.lv
}

ORCID: 0000-0003-3770-7168

\begin{abstract}
New technologies arrive in our daily lives that actualize an urgent issue of preparing of specialists in specific technological fields. Hence, the necessity in providing of relevant education and training opportunities, appropriate digital learning tools, learning management systems and new learning approaches become more pressing. An understanding of students learning needs, their learning experience and activity peculiarities can help a lot to ensure learning process more efficient. And eye tracking technology could be considered as a significant instrument to be used in such studies. This paper provides a brief review on eye tracking technology employed for the findings on an analysis of learning management system user experience, as well as discusses prospective model development directions in this field.
\end{abstract}

Keywords: eye-tracking, gaze, interaction, learning management system, user experience.

\section{Introduction}

People are different, each of them has own specific individuality. This is reflected in their activities, behaviours, individual perceptions of things, affairs and processes. Education professionals and researchers try to understand learners' individual behaviour and activity peculiarities in order to improve the efficiency of the learning process.

These individualities allow researchers to provide appropriate findings with respect to people behaviour patterns looking for personalized adaptive solutions (Zuga et al., 2015). This approach is used in different areas of interest: starting from Web browsing usage experience and e-commerce advertisements till social collaboration and education in digital learning environments. Moreover, in the rapid changing world which brings us new technologies, digital tools and systems, our knowledge society is forced to look for the necessary solutions to improve learning process, which can be achieved by assessing the specific characteristics of students' learning experience within learning management systems (LMS).

In digital learning environments it could be useful to find an effective way to divide learners in groups with regards to their learning activities and learning styles. There were numerous attempts to adapt user learning styles (Danielson, 1997; Gilbert and Han, 
1999), however, the question about the most appropriate classification of learning activities to be reckoned with during modelling of adaptive learning/tutoring solutions, is still not solved yet (Brusilovsky, 2001). Thus, it was concluded that there was the necessity to work on predefining of learning objects depending on students' learning styles and behaviour patterns, bearing in mind that insufficiency of such learning object or their groups might adversely affect adaptive tutoring systems (Graf, 2010).

At the same time, we can find that during recent years information system users' individuality, their learning, social collaboration, trading, internet browsing experience and preferences are being studied also by implication of biosensor technologies and eye tracking tools. Human bio-signal data, in particular gaze data, can give researchers and adaptive IS designers extra new data which would be used to understand users' cognitive issues during exploration of a particular website, as well as an involvement level in the content (McNamara et al., 2015).

Eye tracking tools embedded into adaptive tutoring systems might be considered as the powerful effcious instruments to personalize learning content in accordance to particular student needs, thereby providing effective knowledge delivery and acquisition (Lai et al., 2013). An analysis and proper interpretation of gaze data could help us in developing of new digital learning environment which would promote more efficient learning process. Eye tracking tools and systems provide researchers with a valuable access to IS users' behavioural data (Meisner and Oll, 2019) which, in their turn, give an additional comprehension about cognitive side of human behaviour processes (Ashby et al., 2016).

Previous applications of eye trackers were intended to track and verify user involvement in cognitive processes and the ability to follow the e-materials offered. In addition, it was considered that the student follows all the materials placed on the LMS webpages according to the imaginary plan of the instructional designer. But this is not the case (Jarodzka et al., 2017).

Given that each student has their own unique learning style, subject perception, cognitive abilities, and other behavioral characteristics, it is very important to elucidate the peculiarities of user activities and experience within digital learning environment. To do so, it might be identified several categories that ought to be taken into account when developing e-learning objects, managing and guiding learning process in the digital learning environment: culture, gender, literacy level, very young learners, foreign language acquisition.

The following sections provide an overview of what and how we see on a computer display, how eye trackers can help evaluate learners' experience, including different categories of learners, as well as provide support for necessary improvements within LMS related to e-learning objects and instructional design. The paper also proposes and discusses prospective model research directions in eye tracking application in digital learning environments.

A huge amount of research papers, related to "Eye-tracking AND Learning", are published in scientific journals and conference proceedings. Just in the Web of Science database we can find more than 1900 such sources. But the thing is that majority of them are related to the "learning" issues only. Some of the pure "learning" papers might be linked to this review. However, the aim of the paper is to show the digital learning environment user (i.e. current or potential student) experience, activity and behaviour patterns, which can be assessed by eye trackers. Consequently, research questions related to the learning ought to be addressed to another study. On the other hand, eye tracking technologies, discussed in many other reports, are used also without particular linkage to 
learning process, and such research results are found in many and many articles. The first main criteria for the source inclusion into this review was the relevance of the study/article to the aim of this paper. Next, particular institution's and scientist's work activity impact on developments in eye tracking field was consisred as well. The number of citations in recognizable databases, as well as publications in a high-impact scientific journals and conference proceedings, also enhanced an inclusion of these sources in the reference list.

\section{How and what we see on the computer display}

Even when we think that our vision is stable, that is not correct. During awaking period our eyes are constantly moving, dividing this movement process into saccades (i.e. our eyes are moving and pausing, thus generating a number of saccades; so, it could be said that the saccades are movements between fixations) and fixations (i.e. extremely short time periods, usually 100 to 600 milliseconds, in which our eyes are fixed on a specific area or object). As the fixations usually are very short, our eyes continue movements, giving us a picture of test participant's sight direction and areas of interest (AOI).

People move their eyes in order to see better. This is caused by limitations of human eye where only fovea area can ensure a clear high resolution picture (Carpenter, 1988; Leigh and Zee, 2006). Respectively, our main attention is devoted to areas within foveal vision, which is only less than eight per cent of human visual field (Tobii Technology, 2010). Other major parts of our eyes have parafoveal and peripheral vision which just give us a sense or imagination about things and processes around us, which can provide us only low resolution picture, motion, colour (Rayner, 1998). However, this feature brings a new opportunity for researchers dealing with eye tracking, interface design and user experience studies.

Despite small area of the fovea, other parts of human vision, parafoveal and peripheral vision, provides the person a common general picture of the objects and process flows, which gives a perception of the surroundings, and thereby impacts person's decision process, e.g. choosing of vision object. Therefore, at the same time it might be said that if the eye movements were not fixed at the particular point, it would not be meant that the person did not aware of it (Bergstrom and Schall, 2014).

Eye tracking can provide researchers with clear picture about test participant's eye movements. Heat maps, gaze plots, and gaze video segments are usually employed for gathered gaze data visualisation.

There are three main attributes which compose an understanding about eye movements: location, duration, and movement. The position of the eyes can be mapped on the grid at a specified time between their movements. The duration of fixations could be shown as the dots with variable sizes: as bigger is the dot, the more time was spent by system user to this location. Fig.1 illustrates information system user's eye movements, location and duration of fixations. It should be noted that the gaze data do not necessarily depict user's mind and particular attention. At the moment, eye tracking systems can provide only data about eyes specific fixations which might be an accidental blink of the eyes that the user may not even remember (Bergstrom and Schall, 2014).

Gaze data can provide valuable information about system users interests and cognitive processes. Measuring their activity, behaviour and eye-catching patterns during Web browsing, eye tracking researchers can make some predictions about users' further website visits. An analysis of gathered gaze data can lead for creating or updating 
website design in order to meet website visitors' expectations, and, as a result, make it more efficient and profitable. In the case of learning management systems, it can induce also redesign of e-learning modules and learning objects (location, types, formats, etc.).

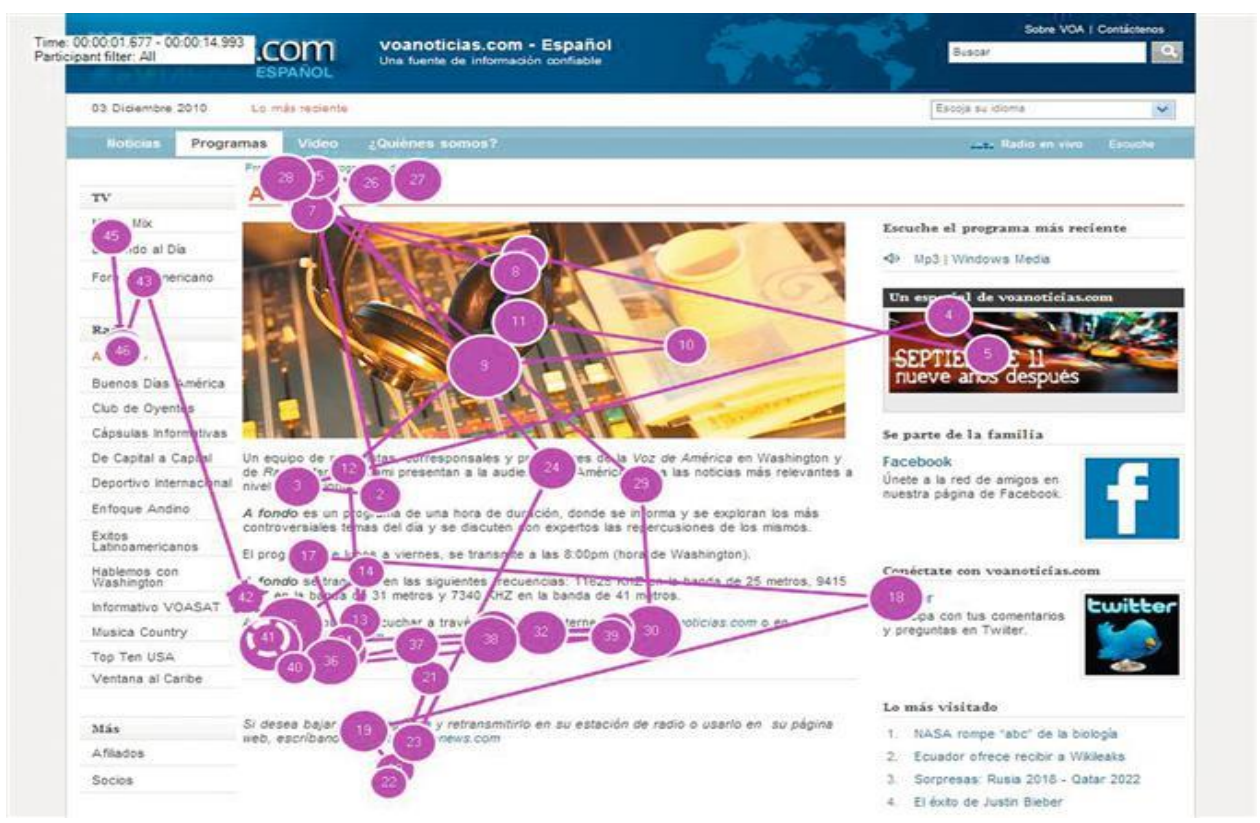

Fig. 1. Mapping of fixations (Bergstrom and Schall, 2014).

One of approaches in order to find out IS users' behaviour patterns and their preferences is based on Visual hierarchy theory which explains website search pathway through a two-phase cognitive process: website scanning phase and inspection phase. During scanning phase, the user scrolls through the page till the entry point is found. Then, within inspection phase the user looks around the entry point for corresponding information. This two-phase process repeats again and again, going through many and many of entry points, where the user has a possibility to search for the information he/she is interested in (Faraday, 2000). It ought to be noted that website characteristics, such as objects type, size, style, colour, location, etc. influence both these phases (Djamasbi and Hall-Phillips, 2014).

The common dominant tendency is that usually users start look at the top and left parts of the website (Faraday, 2000). This tendency has also been called the golden triangle or F-shaped pattern of viewing. It also should be noted that website visitors might lose their attention to some important objects on the bottom and right parts of the website, thereby missing them (Buscher et al., 2009; Nielsen, 2017).

Information system user viewing experience could be illustrated by exploiting heatmaps. Fig. 2 shows an example of the user's website scanning experience, where viewed locations are coloured in different tones: red - the most visited, yellow - moderate attended, blue - infrequently viewed, and grey - have not interested in (Nielsen, 2006). 

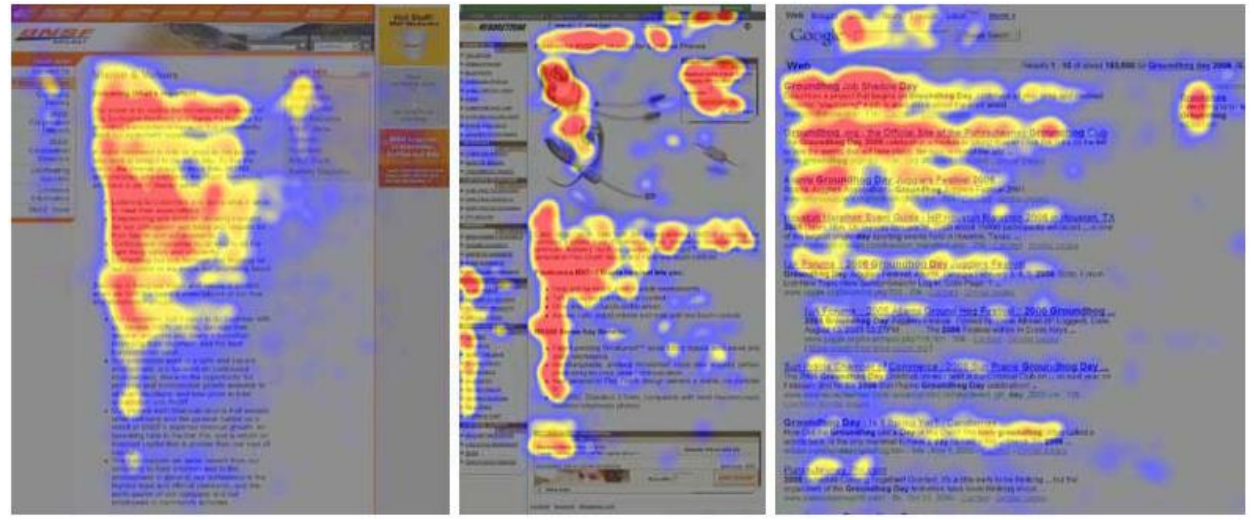

Fig. 2. Website scan-paths - gaze heat-maps (Nielsen, 2006).

\section{Eye-tracking technology in digital learning environments}

\subsection{Types of eye tracking systems used in digital learning environments}

Basic function of eye tracking systems is to find the link between the system user and the system interface by measuring user's interest in the particular content. They track eye position and movement in real time in order to determine and evaluate system user's visual attention. Human-computer interactions produce a big amount of data about information system user's activities within the system. Learning analytics can provide necessary conclusions regarding user's preferences and habits during computer interaction sessions, including number of log-files, type of used files, and so on. More and more popular eye tracking systems allow monitoring and analysing users' preferences with regards to digital content search and interface preferences. Eye tracking has significant advantages over more traditional experimental techniques: it might be done without a secondary task, such as a button press in self-paced reading, allowing for natural reading or viewing; besides, it provides a very rich record of gaze behaviour which would represented by various visual forms (Raschke et al, 2014). Eye tracking systems are also used to investigate human cognition processes (Clay et al., 2019).

Speaking about prospective ideal eye tracking system, it would be useful to specify appropriate requirements considered in previous studies (Boff et al., 1986; Scott and Findlay, 1993; Glenstrup, 1995). However, we ought to bear in mind that not of all existing technologies can completely support them, e.g. such questions might be raised regarding an accuracy, resolution, detection of even minor eyes movements, free unattached user access to the display and unobstructed field of view, improvements of real-time response, competitively wide dynamic range for eye position and eye velocity, and so on (Mohamed et al., 2008).

Eye movement activity assessment devices usually are divided into three main types: computer (laptop) screen mount devices (also called - desktop eye trackers), eye movement activity assessment glasses (also called - mobile eye trackers), and eye trackers embedded within virtual reality headsets (Farnsworth, 2018). In the case of onscreen equipment, the person must be in front of the computer screen and the equipment monitors the movements of the user's eyes, assessing their activity and interaction with 
the content displayed on the screen. Although there are limits to the amount of head and eye rotation that modern eye goggles have, it does not interfere with the on-screen monitoring of user attention points, providing an accurate overview of the person's interests displayed. In the educational process, it would be important to identify those learning objects which are more attractive for the students, and where the text, video, image or exercise units would be better placed on the screen.

Nowadays the most widely employed eye trackers use video-based design. At the same time the market holds out eye trackers that integrate ordinary low-resolution webcams and use no infrared light. Video-based eye tracking relies on off-the-shelf components and video cameras, and therefore can be used for developing eye-aware or attentive user interfaces that do not strictly require accurate point of gaze tracking (Hansen and Pece, 2005; Hansen and Majaranta, 2012). While these systems are less expensive and seem to be an ideal solution for smaller budgets, the lack of a controlled light source along with a rather weak camera performance will reduce measurement accuracy significantly (Blascheck et al., 2014, 2017). According to scientific publications indexed in SCOPUS, IEEE Xplore Digital Library, ScienceDirect, ACM Digital Library, and others, it might be said that following main eye tracking systems are academically approved: Tobii Technology, myGaze, SensoMotoric Instruments (SMI), Eyetech Digital Systems, Smart Eye, TheEye Tribe, and GazePoint. They all offer wide range of eye tracking possibilities; however, only SMI provides completely functioning system, but in the case of Tobii and myGaze a customer is asked to obtain Morae software allowing to record, observe, and analyse usability studies, focus groups, field research, and product testing. The program allows watching interactions remotely, taking notes, analysing results, and instantly sharing observation results with colleagues and other students.

Several universities, industrial leaders in eye tracking system development and appropriate research institutions have an expertise in eye tracking in their labs, but are less knowledgeable about education-focused research. They focus mainly on studies in neuroscience, marketing, visualization research (Toker et al., 2013), as well an accuracy in medical diagnostics graph reading (Davies et al., 2016) and Webpage usability (Eraslan et al., 2016). Despite studies where gaze data of both group and individual users were collected also in virtual learning environment (Brajnik et al., 2016; Kaššák et al., 2016), there are no sufficient signs and clear directions on how to apply such data in order to enhance users' self-discipline, improve learning outcomes, learners' engagement and motivation.

It might be also noted that the belonging to different nations and cultures have an impact on information system users' behaviour traits. At the same time, the studies, fulfilled in human computer interaction field with respect to cultural and gender aspects, ought to be continued and extended.

\subsection{Eye tracking technology: cultural impact}

Cultural issues play significant role in studies on human-computer interaction (HCI). Regarding cross-cultural issues, the book "International User Interfaces" (Fernandes, 1995) remains the classical one for many researchers till now.

Research activities on possible impact of differrent cultures on particular nation and culture behaviour features during interaction with digital systems are usually implemented by multinational research teams (Heimgärtner, 2007; Schoper and Heimgärtner, 2013). It should be noted that working in multinational environment shows 
significant cultural behaviour differences which are based on different, and sometimes totally distinctive, thinking process, e.g. abstract thinking ability differences, reporting deadlines, hierarchy levels, willingness to work on the project only if its results bring tangible benefits to the customers, and so on (Hofstede and Hofstede, 2010). This all influences HCI project team's common outcomes. At the same time, it allows to get know persons' behaviour features and cultural individualities (Heimgärtner, 2013; Heimgärtner and Solanki, 2014). It could be said that this aspect not only may cause delays in some cases, but also could help to develop products which suit particular nation and culture the most.

Information system service and product developers also ought to think about their customers, bearing in mind cultural, national and gender diversity issues (Plocher et al., 2012). Product's user interface engineering process is culturally influenced (Honold, 1999); so, user interfaces ought to be developed depending on particular customer group cultural and behavioural traits (Röse, 2005; Heimgärtner, 2014). In order to succeed, cross-cultural user interface engineers ought to know at least basic proved statements and principles on cultural diversity issues (Thomas et al., 2010).

An influence of culture on product usability, besides cultural imprints, such as religion, prosperity, gender attitude, education, age proportion, customs, etc. (Honold, 1999), could be characterized by several key influencing parameters, e.g. levels of individualism or collectivism in particular nation or cultural group, masculinity, distance of power, indulgence, pragmatism, avoidance of uncertainty, etc. (Hofstede and Hofstede, 2010).

These cultural differences are really immense and ought to be considered during information system user interface designing. Some of cultural models could be more influential (for example, individualism vs. collectivism), others - less (Vöhringer-Kuhnt, 2002). However, irrespective of whether these criteria are more or less influential, interactive information systems should suit not only overall usability requirements but also take into consideration cultural aspects (Heimgartner, 2013).

Interesting eye tracking studies were performed recently, where attentional and communicative parameters of the United Kingdom and Hong Kong teachers were compared. One of the aims was to investigate how teachers from different countries (i.e. cultures) behave while giving lessons and providing answers and information to students, as well as asking students. Inter alia, it was found that experienced teachers, regardless of culture, have shown prioritisation of learners, however regarding communication processes Hong Kong teachers have shown prioritisation of instructional area, but UK teachers - non-instructional ones (McIntyre et al., 2019).

Cross-cultural findings in $\mathrm{HCI}$ have been continuing in further projects emphasising an importance of the design of User Interfaces (UI) and User Experience (UX) (Heimgärtner et al., 2018).

\subsection{Eye tracking technology: gender considerations}

Partially gender issue might be seen as the continuation of cultural imprint diversity influence on human-computer interaction (HCI). Scientists are very cautious regarding HCI research on gender differences. Though, some findings could be found and discussed. Eye tracking tools are also used in these projects.

Rather early studies already investigated eye movement differences between genders in different age groups. It was found that eye movements differ by gender only in the adult phase (Miyahira et al., 2000). 
In 2014 Ministry of Science and Technology, Taiwan, disseminated research results regarding gender differences in epistemic beliefs and the reading process. Students' (both females and males) gaze data were used to record their science-text reading process. It was found that female students showed less mental effort in understanding scientific explanations. Male students had more interests in studying scientific matters. On the other hand, female students preferred to attend more data and micro-view graphic learning themes. It was also found that female students, rather than male ones, showed better results on tasks related to processing of textual information (Yang et al., 2016).

Vilnius University conducted the study on learners' book cover colour preferences. It was observed that the book cover colour influenced such choice. Research results have shown that women in the age group from 18 till 35 preferred cool colours; however, in older women's age group this preference gradually disappeared. In turn, men in the age group after 56 preferred warm cover colours. There was not observed the choice of such a warm colour in the younger men group. Researchers have also found that in women's group there was a positive correlation between the time spent for choosing a book with preferred cover colour and time looking at this book; in turn, no any correlations were found in men's group (Gudinavičius and Šuminas, 2018). These findings could suggest instructional designers to develop frames and graphics of digital learning objects coloured in tones which are accepted by particular learners' group.

\subsection{Eye tracking technology: very young readers}

How often we imagine reading material acquisition and comprehension process among young readers? Do we think enough about complexity of materials for reading, even in the cases of rather basic, entry level reading texts? What could be said about readers' progress?

In many studies researchers assume that all learning management system users read the text similarly, so, text perception is seemed as the similar letter and word recognition process for all (Reichle et al., 2003; Engbert et al., 2002; Reichle et al., 1998). However, it could be noted that there are differences in reading skills among people and this aspect should be taken into consideration in order to adjust models of eye movement control in reading (Ashby et al., 2005).

Children's reading, perception, comprehension, behaviour and attitude issues have been discussed in a number of research papers on psychology and cognition. They ought to be taken into account while performing eye tracking research. One of the most comprehensive studies on the textual material perception and reading issues occurring at the first reading steps could be reached in Keith Rayner and other colleagues' publications (for example, Rayner et al., 2012; Rayner et al., 2006; Rayner, 2009; 1998; White and Liversedge, 2006).

The length and complexity of words affect eye fixation (Rayner, 1998). Long words require more time from a young reader (Rayner et al., 2012) and might be omitted by them (White and Liversedge, 2004; Rayner et al., 1996). It was also found that refixations depend on linguistic characteristics of the word (White and Liversedge, 2006), so, the learners returned to rather complex words as more trained and experienced they became. It could be also noted that children's reading experience and reading skills improvement led to shifting of their eyes concentration from separate letters to the whole word and the sentence (Rayner et al., 2012).

Parafoveal visual information is also used by very young readers. This guides children's saccades during reading process, and no any significant differences from adult 
readers is found despite some limitations of children's parafoveal vision (Blythe and Joseph, 2011).

In studies on reading fluency, both oral and silent, oculomotor data confirmed that all children achieved learning, i.e. reading speed, progress from the first testing time to the second one. Moreover, better reading progress was noticed among children who had worse results during initial test. Besides, it was also found that the oral reading required more mental concentration in order to understand reading material (Vorstius et al., 2015).

\subsection{Eye tracking technology: people with low literacy}

Low literacy abilities mean that such persons have particular difficulties with recognizing of words in text sentences. This impact their abilities to learn due to required much longer time to read and understand textual and graphical materials. Besides, the text they read may be misinterpreted (White, 2003). On the other hand, low literacy should be considered not only as the reading problem. This group of population also have problems to perceiving mutual information. They may have thinking and problem solving ways which differ from other people approaches (Canadian Association of Chiefs of Police, 2008).

During usability tests their participants might be asked to give feedback comments on processes doing by them. This approach, called "think-aloud" method, provides valuable information for interface developers and instructional designers. Despite undeniable benefits, the method has also some constraints. For example, it overloads test takers by asking them not only fulfil particular tests but also describe mutually things they are doing and thinking (Brinkman, 1992). Moreover, by forcing test takers "think aloud", this may change their task accomplishment pathway. As a result, activity and behavioural patterns of the participants might be changed, but this is not what the researchers do want to get know (Nagle and Zietlow, 2012). Bearing in mind that cognitive impairments also affect reading abilities, it might be said that "think aloud" methods becomes less useful for people with cognitive impairments (Johnstone, 2006).

On the other hand, eye trackers and gaze data can display the picture of test taker's real usability experience within information system (IS) and task accomplishment steps. Eye tracking does not set additional cognitive pressure, thus, it could be considered as the more useful instrument for usability test implementation among persons with low literacy skills (Colter and Summers, 2014). It might be suggested that combination of eye tracking and other human bio-signal processing systems can provide more efficient instruments and data while testing persons with other types of cognitive impairments.

Very often persons with low literacy are unable to recognize themselves being difficult to read and correctly perceive the information. Moreover, it was ascertained that the persons try to mislead other people: colleagues, friends and even relatives (Kirsch, 2002). Eye tracking tools can help educational practitioners to identify such persons and give them appropriate teaching support.

It is obvious that complex textual and graphical structures in many cases may impact comprehension and perceptual abilities among skilled readers (Romano and Chen, 2011). Hence, complicated text structure and unusual arrangement may even despair persons with low literacy. It is underlined that improved IS interfaces and organized, easy-to-read structured text can assist not only skilled readers but also low literacy readers, giving them giving them an opportunity to understand particular material faster 
and better, accordingly, getting pleasure and satisfaction from reading activities (Jarrett, 2012).

Eye tracking can help to investigate readers' behaviour during reading, possible obstacles, as well the things which provokes reading difficulties. It was found that due to difficulties in correct understanding of textual materials persons with low literacy skills spend more effort on reading than necessary. To save this category of people, it is suggested to prepare digital learning materials clear and easy understandable as much as possible (Summers and Summers, 2004). Next, low literacy skilled persons often try to avoid reading all textual information because of additional cognitive efforts needed for such task (Summers et al., 2006). If the gaze data warn that particular IS user skips the text, this information will be used to improve product usability and reading efficiency by simplifying text and reorganizing text structure (Doak et al., 1996).

Eye tracking researchers ought to keep in mind another strategy used by persons with low literacy skills during reading. The thing is that this group of people try to avoid cognitive efforts what is achieved by accepting the first plausible answer which they found and stop reading further; it does not depend on whether this answer is right and complete or not (Krosnick, 1991, Summers and Summers, 2005). It could be recommended to instructional and user experience designers to enable first possible answers in textual materials, as the most suitable and understandable for persons with low literacy skills (Colter and Summers, 2014).

Eye tracking tools are very useful also to assess which parts of an interface are more distracting to low literacy persons. By adding any element to the webpage (it might be learning object onto learning management system or advertisement banner onto online shop website, etc.) in different forms of static or moving textual, graphical or audiovisual information, system user is experiencing a problem with competing of his/her attention to each such element. For the people with low literacy noted competition for their attention is damaging - they have problems with reading rather simple texts, but a complex structure with anew changing elements really can raise the distraction (Mayer, 2009). Eye tracking can help to determine which particular objects on the screen attract IS user's attention the most. Necessary changes in interface design, making pictures and textual materials simpler, can reduce cognitive effort of finding appropriate learning objects and other reading materials. Hence, by doing this we can enhance reading and learning experience for the persons with low literacy (Colter and Summers, 2014).

Pursuant to above noted studies, it could be concluded that persons with low literacy often read almost each word in the text, they need more and longer attention devoted on text; re-reading of text and returning back to interface elements is quite often issue among them. Besides, sometimes they try to escape from reading of the whole text, skipping some words in sentences, whole sentences and even paragraphs, as the reading requires extra attention and cognitive exertion. Stopping reading after getting the first probably more or less satisfying answer is an another issue. Complexity in interface design, almost each change in textual information disposition on the computer screen distracts persons with low literacy, as these changes require from them additional cognitive efforts (Colter and Summers, 2014).

\subsection{Eye tracking technology: foreign language text acquisition}

Interesting study results were displayed by researchers from Taiwan who investigated non-native speaking learners' trust levels on audio recording of English textual materials for perceiving, in particular, a correlation between the level of perceiving information 
and possible phonological recoding interference. They did not find significant impact of reading conditions (among them - text complexity) on comprehension. However, an increased eye movement activity during reading process was noticed (Guan and Hsu, 2015).

Another study was dedicated to recognize how differences in language skills can impact eye movements of kids. Researchers found the correlation with strong significant relationships between children language skills and their eye movements during text reading way their eyes go through a text. Inter alia, it was confirmed that children with good comprehension made less re-fixations, produced longer saccades, and reading time was shorter in comparison to kids with poor comprehension (Mancheva et al., 2015).

Facebook introduced an algorithm personalised to user's experience within this social collaboration platform. Four main parameters were taken into account to determine particular user interests and engagement level: posting author, posting time, content type, and interactions with the post (Constine, 2016). The issue of the level of interactivity remained unresolved in the cases when involving people with different language skills.

In another study it was found that foreign language knowledge level directly influences students' attention and engagement in collaborative working environment. English speaking participants were much more involved in posting comments and contributions to the sites with graphics and text in English or one of the Western foreign languages, especially in the cases when they were able to give their inputs in English. In contrary, images and their descriptions in Asian languages did not attract an attention of non-native speakers. It is suggested that modern online translating services could help both teachers and learners to interact more actively in collaborative digital learning environments (Lim and Fussel, 2017).

\subsection{Eye tracking technology: assisting academia}

Eye tracking tools and systems have more and more widely used in educational studies, giving an opportunity to get deeper insight of learners' digital cognitive activities. Specific eye tracking implementation and gaze data analysis conferences and working groups are set to find sophisticated, more powerful solutions enhancing learning efficiency (e.g. EARLI SIG-27, 2017; Jarodzka et al., 2017; Oliva et al. 2017; Lai et al., 2013; She and Chen, 2009; Yang et al., 2013; Anderson et al., 2014, etc.).

Eye tracking researchers and developers have shown that gaze data could give necessary futuristic vision and directions on development of multimedia learning objects, methods and processes (Alemdag \& Cagiltay, 2018; Hyönä, 2010; Liu et al., 2011; Slykhuis et al, 2005; Yang et al., 2013, etc.). Eye trackers are also successfully used to increase an efficiency of the learning process for students in engineering and software development field, hence, improving their specific knowledge and skills (Turenko et al., 2019; Yenigalla et al., 2016; Sharafi et al., 2015; Jbara and Feitelson, 2015).

A broad overview on fulfilled studies related to eye tracking techniques and applications is provided by Lai and colleagues (Lai et al., 2013). They give an evaluation of existing research directions in the eye tracking and particular fields, such as psychology, information processing, cognition, HCI, complex learning contexts, and so on. Proposed directions for further work include adaptivity approach which would provide students with personalized learning path within LMS equipped with eye trackers (Lai et al., 2013). 
Eye tracking tools are also considered as a prospective interface tools embedded within existing learning management systems or adaptive tutoring systems. Eye trackers can measure changes in learner's cognitive load, accordingly, providing useful data to enhance learning experience (Rosch and Vogel-Walcutt, 2013).

As long as human behaviour, psychology and educational researchers have been working on finding out learners' activities, behaves, attitudes, and engagement level, the eye tracking technology experts and enthusiasts are looking for new solutions. One of the most important challenges is to enhance education efficiency, keep learners engaged and interested in learning process, make preventive steps to reduce drop-outs as much as possible. It also could lead to adaptation of the offered learning content or development of adaptive tutoring / instructional system.

To do so, scientists are focused on identifying what types of learning objects (LO) the students are focusing on; which LO attracts them more and why, which interface layouts impact learning process, and so on. The majority of learning materials students obtain via eyes. Therefore, an awareness about specifics of the information perception and knowledge acquisition process is so important. In recent years, eye tracking has been playing more active role in learning management system users experience and e-learning process research (Holmqvist et al., 2011).

Human-computer interaction (HCI) and interface usability studies provide significant amount of users' activity data while working in digital environment (Jacob and Karn, 2003). The one of the most studied areas in applied eye tracking research is linguistics, reading skills development (Rayner, 1998; 2009; White and Liversedge, 2004; 2006; Rayner et al., 2012).

Gaze data can give important information about usability of learning management system interfaces, for example, Moodle e-platform usability eye tracking tests (Rakoczi, 2010), as well as LO within them, propose appropriate recommendations regarding necessary steps to improve instructional design, for example, in testing of two Romanian e-learning platforms (Chivu et al., 2018). Eye tracking technology can give valuable data to evaluate also students learning activity patterns, for example, regarding the number of learners in groups while acting in searching, viewing and preparation activities (Nakayama and Shimizu, 2006).

\subsection{Awareness of cognitive load in technology enhanced learning (TEL)}

Gaze data already are used in almost all spheres of educational science research. However, this applied eye tracking research field is relatively young. It is largely grounded on the theories employed in educational science research.

One of the most comprehensive eye tracking studies in educational science research are fulfilled by Jarodzka, Holmqvist and Gruber (2017). They provide a broad view of the two essential eye tracking research fields in education, i.e. instructional design adapting the environment to the learner's abilities, and expertise development scaffolding the learner to the environment. There they take into account also theories of fundamental research on cognition and perception, as well suggesting eye movement modelling training method (Jarodzka et al., 2017).

In order to bring together both abovementioned research areas, the Eye Movement Modelling Example (EMME) was proposed, assisting novices to learn, getting assistance from a model, i.e. an expert. The aim of the EMME is to investigate, how "visual expertise could be trained with the help of instructional videos of real-world tasks that are explained by experts in the field" (Jarodzka et al., 2017). Expert's eye movements 
are recorded while solving a task and that video recording is displayed to the novices (Van Gog et al., 2009).

EMME could be very useful in visual task training. At the same time, researchers ought to keep in mind that novices are often already overwhelmed with information-rich material that forms the basis of visual tasks. Adding an eye movement display on top of that, is likely to overwhelm them. As an alternative solution is to display the model's eye movements by reducing existing information on videos (Dorr et al., 2008; Nyström and Holmqvist, 2008).

EMME has displayed significant differences between experts and novices. It was concluded (Jarodzka et al., 2017) that the expectations about novices' learning or working activities level would not be at the similar extent as for experts. EMME researchers in their findings used a systematic way to make the expert model act more didactical. Two considerations were taken into account: firstly, the models in EMME were always not only experts in particular domains, but also highly experienced in teaching this domain, knowing very well possible difficulties which students could face in fulfilling these tasks, as well optimal the most efficient explanation methods to the learners. Secondly, EMME researchers used a specific recording procedure in order to make sure that the EMME videos were of high quality: it was succeeded by ensuring of the close relation of the voice and the eye movements of the models, which researchers first show them the task itself (for example, video recording, other issues which would be needed to classify). Only after test participants are familiar with specific task, the recording might be started.

Next, in order to shift models' focuses from the task to the novice recipient, they evaluate their own recordings based on several questions: (a) Will a student know what each term means? (b) Is the task explained in comprehensible enough terms for students? (c) Is it explained enough detailed? (d) Are the all instructional materials, that a student needs, contained? (e) Are the all contained information really important? These questions have appeared to improve written communication of experts to novices (Jucks et al., 2007). And finally, the models are enabled to revise their recordings in a case of necessity.

EMME approach was already employed in several other studies by Jarodzka's team, for example, in the domain of diagnosing epileptic seizures in infants (Jarodzka et al., 2012), in classification training of the locomotion patterns of reef fish (Jarodzka et al., 2013). Other scientists have also used similar training approaches: for visual tasks, which require prior obtained knowledge (Litchfield and Ball, 2011; Mason et al., 2013; Skuballa et al., 2015), for expertise tasks (Leff et al., 2015; McNamara et al., 2012; Sridharan et al., 2012), for problem solving in dyadic interaction (Cherubini et al., 2010). Nevertheless, these studies did not investigate whether learning occurred or not.

It is worth mentioning about some studies which reported that EMME approach had no or even detrimental effects (Skuballa et al., 2012; Van Gog et al., 2009). Respectively, it could be useful to rephrase the research question, which "is not whether EMME does foster the performance of visual tasks (or even visual expertise), but rather, under which circumstances in does so" (Jarodzka et al., 2017).

\subsection{Eye tracking technology and other bio-signal processing systems}

Eye tracking technology is used to observe and record individual's eye movements through a special hardware and software. There are different types of the hardware: remote, non-intrusive (i.e. screen-based eye-trackers), or head-mounted, which are more 
intrusive, but are the most accurate and give total freedom of movements to participants, for example, glasses that have an inbuilt eye tracker (Asteriadis et al., 2009). The most modern eye tracking systems record data through a small, high-resolution camera that uses infrared to analyse corneal reflection (iMotions, 2018).

Most eye-trackers focus on the two main eye movements: saccades and fixations. Obviously, there are much more eye movements, nevertheless, these two are taken into account the most. Saccades are voluntarily rapid eye movements, lasting between 150$600 \mathrm{~ms}$ and happen between fixations. Fixations are miniature eye movements that last between 10-100 ms and during which the eye almost holds still. Fixations represent approximately 90 per cent of the person's viewing time (Duchowski, 2017), being also the moments when visual information can be processed.

During saccades, little or no information could be processed. This challenge could be considered as an important hidden opportunity to propose new research directions and make further findings in the field of information system user experience.

Information system user experience gaze data could serve as an indicator of attention, and, respectively, attractiveness of information presented at the webpage. For example, the number of fixations is considered to indicate how much attention IS user pays to certain stimuli (Holmqvist et al., 2012). According to the "eye-mind assumption", the information that is seen is processed at a cognitive level; in other words, it could be said: "what people are observing, so that they are also thinking" (Just and Carpenter, 1980).

At the same time, it must be noted that some findings show that the eyes do not always mirror cognitive processes (Anderson et al., 2004; Ashby et al., 2016). Hence, inferences should not be based only on data collected through eye movements. Besides information about attention, gaze data can reveal information about emotional states of the learner. For example, there are studies which show that highly arousing positive or negative stimuli lead to a significantly larger pupil size, when compared to neutral or medium arousing stimuli (Partala et al., 2000). It might be recommended to confront gaze data with the data gathered through other system and tools, for example, electroencephalography, electromyography, electrocardiogram, electro-dermal activity, galvanic skin response, face recognition systems, facial expression analysis, etc. (iMotions, 2019).

\section{Discussion}

When designing an adaptive e-learning system based on gaze data analysis, certain factors should be accounted for. Firstly, there are studies that demonstrated that eye movements act as a function of culture (Chua et al., 2005; Kelly et al., 2010). Secondly, gender is also believed to influence eye movement strategies (Mueller et al., 2008). Another study showed that eye movements differ by gender only in the adult phase (Miyahira et al., 2000). Thirdly, eye movements are influenced by cognitive styles. Cognitive styles are "an individual's typical or habitual mode of problem solving, thinking, perceiving or remembering" (Tsianos et al., 2009). In other words, cognitive style shows how an individual perceives and processes the data.

Investigating recent findings in the field of eye tracking technology and systems, the further conceptual model development directions in the educational settings could be considered as follow:

Taking into account that during saccades little or no information could be processed, to find solutions regarding interpretation of gathered information about performed 
saccades. This could reveal a new understanding of user activities and behaviour patterns in UX research.

To find out which e-learning object type engages the student the most, including colour and tone shades, size, layout, on-screen appearance, sequencing, banners, etc. These studies might involve gender difference issues.

To study learner's behaviour patterns, activities, psycho-emotional state and their changes, employing both eye tracking system and other biosensor equipment, e.g. electroencephalography, allowing a more accurate determination of the user's excitement or alertness level during knowledge acquisition in digital environment.

Taking into account achievements in human-computer interaction research field regarding cultural differences, it might be proposed research direction which would support these findings employing eye tracking technology and analysis of gaze data. Research on getting know persons' behaviour traits depending on cultural individualities could help to create digital learning objects which suit particular nation and/or culture representatives the most.

To measure the level of student fatigue and a certain degree of learning apathy or boredom. It facilitates an assessment of students' involvement in the learning process, keeping them "hot", thus providing an opportunity to improve the e-learning course and instructional design (analysing user individuality, behavioural activity patterns, fatigue detection, and appropriate metrics employment, etc.).

To consider the issue of protecting users' eyes while working with displays, making sure that the information system user does not overdo his/her eyes (parameters, such as, time without blinking, and so on, could be determined and analysed), as it can lead to eye fatigue, dry eyes and inflammation, which can negatively affect the vision and perception of the subject within e-learning environment. It could be solved considering some kind of a reminder on the computer or smartphone screen (display) which would float automatically if it detects, that the user has not blinked for a certain amount of time. Moreover, proposed research direction might be considered to be given in combining with the previous research direction proposal regarding fatigue and boredom issues.

To explore the use of eye tracking in virtual and augmented reality (VR/AR) eenvironments: identification and evaluation of learner's attention (gaze, AOI, POI, direction) and responsiveness to the changes in the synthetic e-environment; development of responsive recommendations for learning objects (LO) and environmental design. Enrichment of After Action Review (AAR) by adding gaze data results to AAR evaluation after each military training and exercise activity, hereby enhancing determination quality of the lessons identified and lessons learned (LI \& LL).

Assessment of student learning progress using adapted learning object (LO) curves in conjunction with the eye tracking technology. The thing is that learners' group might be relatively divided in several parts, depending on knowledge acquisition results. This would compose imaginable form of the triangle: one corner will be provided for the students who learn easily (i.e. the best performers), the second corner would collect moderate performers, those who go step by step in knowledge acquisition, and the third corner would be used to allocate the worst performers. It would be important to monitor learners' performance on the regular basis at all educational modules. Each individual learner's corner entity can move from one corner to others, depending on student's learning progress and changes in their ability to acquire LO. Gaze data would display possible student's performance correlations in comparison with the analysis data obtained from adapted LO curves. 
From instructional design perspective it could be useful to find the ways for adapting digital learning environment, i.e. learning management systems, to the learner's abilities which suit their needs the most. That might include findings related to learning content improvement, possible adaptive solutions of preferred content types, direct or linked location on the display and accessibility.

Determination of user experience (UX), i.e. learner's attention and engagement into learning process could play significant role here. Information system user's learning preferences, activities, and behaviour patterns could lead not only to knowing about learner's engagement level, interest in particular content (i.e. learning object), attention, but also might signalize about possible boredom. IS user's activities ought to be observed, appropriate gaze and other learning analytics data gathered, processed and analysed at each step of the learning process (module). These data would allow better understand learner's study progress, any problems with learning content acquisition. They are also aimed to render necessary educational assistance to particular learner if he/she has faced with any problem related to learning (both pedagogical and technical ones).

\section{Conclusions}

Eye tracking systems and their generated gaze data can provide researchers with a clear picture about test participant's eye movements. Heat maps, gaze plots, and gaze video segments are usually employed to visualise user experience gathered by eye tracking technology. Three main attributes compose an understanding about eye movements: location, duration, and movement.

An important thing is that eye tracking system records all de facto eye movements, including unknowingly made. This feature makes it possible to analyse possible side effects which impact information system user's decisions.

Information system user experience could be effectively studied by involving visual hierarchy theory which explains website search pathway through a two-phase cognitive process: website scanning phase, i.e. corresponding to the exploratory search behaviour type, and inspection phase, i.e. corresponding to the goal-directed behaviour type.

Interactive information systems designers ought to take into account not only general design and implementation criteria, but also particular cultural issues. Cultural differences, such as masculinity, distance of power, indulgence, pragmatism, avoidance of uncertainty, etc., are really immense and ought to be considered during IS user interface designing. It could be also noted that the F-shaped pattern of viewing is considered as the most frequent dominant pattern, however, it depends on cultural traits regarding text reading direction.

When designing an adaptive e-learning management system based on eye movement research, certain features should be accounted for. Proper consideration of factors, such as culture, gender, age, literacy, and cognitive styles, can result in development of efficacious digital learning environment. However, succeed advantages might have also possible side effects, e.g. limitation of these systems in distribution and application area.

During learning process, we mostly perceive the subject matter through the eyes. Because learning process is based on where, what, for how long, and in what order we are looking at, an eye tracker can help determine that. By knowing the characteristics of users' behaviour and activities, i.e. learning preferences, we can improve instructional design in technology enhanced learning. 
In order to improve an effectiveness of information system user experience research, it would be useful to consider an application of eye tracking technology in combination with other biosensor systems. Additional tools and analytical data might explore hidden patterns in user behaviour and activities. Especially it ought to be kept in mind while working on development of adaptive tutoring systems.

\section{List of abbreviations used}

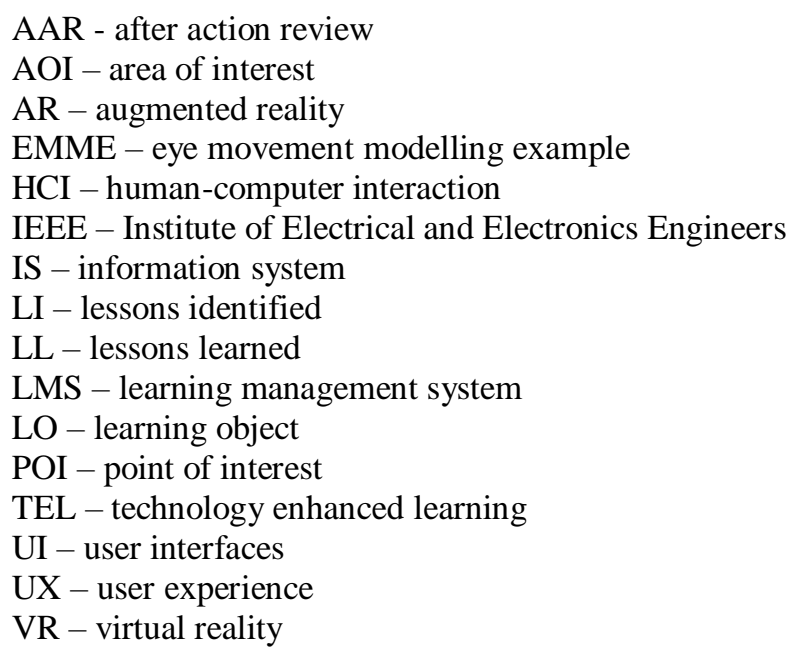

\section{Acknowledgements}

This work has been supported by the European Regional Development Fund within the Activity 1.1.1.2 "Post-doctoral Research Aid" of the Specific Aid Objective 1.1.1 "To increase the research and innovative capacity of scientific institutions of Latvia and the ability to attract external financing, investing in human resources and infrastructure" of the Operational Programme "Growth and Employment" (No.1.1.1.2/VIAA/1/16/042).

\section{References}

Alemdag, E., Cagiltay, K. (2018). A systematic review of eye tracking research on multimedia learning. Computers and Education, 125, 413-428.

Anderson, J.R., Bothell, D., Douglass, S. (2004). Eye movements do not reflect retrieval processes: Limits of the eye-mind hypothesis. Psychological Science, 15(4), 225-231.

Anderson, O.R., Love, B.C., Tsai, M.J. (2014). Neuroscience perspectives for science and mathematics learning in technology-enhanced learning environment. International Journal of Science and Mathematics Education, 12(3), 467-474.

Ashby, J., Johnson, J.G., Krajbich, I., Wedel, M. (2016). Applications and innovations of eyemovement research in judgment and decision making. Journal of Behavioral Decision Making, 29(2-3), 96-102.

Ashby, J., Rayner, K., Clifton Jr., C. (2005). Eye movements of highly skilled and average readers: Differential effects of frequency and predictability. The Quarterly Journal of Experimental Psychology, 58A (6), 1065-1086. 
Asteriadis, S., Tzouveli, P., Karpouzis, K., Kollias, S. (2009). Estimation of behavioral user state based on eye gaze and head pose-application in an e-learning environment. Multimedia Tools and Applications, 41(3), 469-493.

Bergstrom, J.C.R., Schall, A.J. (2014). Introduction to Eye Tracking. In Book: Eye Tracking in User Experience Design (eds. by Bergstrom, J.C.R. and Schall, A. J.): Ch. 1, pp. 3-26. Elsevier: Morgan Kaufman, ISBN 9780124081383.

Blascheck, T., Kurzhals, K., Raschke, M., Burch, M., Weiskopf, D., Ertl, T. (2014). State-of-theArt of Visualization for Eye Tracking Data. In: Borgo, R., Maciejewski, R., Viola, I. (Ed.): Eurographics Conference on Visualization (EuroVis), 1-20.

Blascheck, T., Kurzhals, K., Raschke, M., Burch, M., Weiskopf, D., Ertl, T. (2017). Visualization of Eye Tracking Data: A Taxonomy and Survey. Computer Graphics Forum, 36(8), 260 284.

Blythe, H.I., Joseph, H.S.S.L. (2011). Children's eye movements during reading. In Liversedge, S. P., Gilchrist, I.D., Everling, S. (Eds.), Oxford library of psychology. The Oxford handbook of eye movements (pp. 643-662). Oxford University Press.

Boff, L., Kaufman, K.R., Thomas, J.P. (1986). Handbook of Perception and Human Performance. New York, Wiley-Interscience.

Brajnik, G., Vigo, M., Yesilada, Y., Harper, S. (2016). Group versus Individual Web Accessibility Evaluations: Effects with Novice Evaluators. Interacting with Computers, 28(6), 843-861.

Brinkman, J.A. (1992). Methodological problems when determining verbal protocol accuracy empirically. EUT - BDK report. Dept. of Industrial Engineering and Management Science, Vol. 52, Technische Universiteit Eindhoven.

Brusilovsky, P. (2001). Adaptive Hypermedia. User Modeling and User-Adapted Interaction, 11(1-2), 87-110.

Buscher, G., Cutrell, E., Morris, M.R. (2009). What do you see when you're surfing? Using eye tracking to predict salient regions of web pages. In: Proceedings of the SIGCHI, Conference on Human Factors in Computing Systems. Boston, MA: ACM; 21-30.

Canadian Association of Chiefs of Police (2008). Identifying People Who Have Low Literacy. In: Target Crime with Literacy. Ch.4, Available at: http://policeabc.ca/files/factsheets englishPDFs/Ch04Fact Sheet $01 . p d f$

Carpenter, R.H.S. (1988). Movements of the eyes (2nd rev. \& enlarged ed.). London, England: Pion Limited.

Cherubini, M., Nüssli, M.A., Dillenbourg, P. (2010). This is it! Indicating and looking in collaborative work at distance. Journal of Eye Movement Research, 3(5), 1-20.

Chivu, R.-G., Turlacu, L.-M., Stoica, I., Radu, A.-V. (2018). Identifying the effectiveness of elearning platforms among students using Eye-Tracking technology. The 4th International Conference on Higher Education Advances (HEAd'18), Editorial Universitat Politècnica de València, 621-628.

Chua, H.F., Boland, J.E., Nisbett, R.E. (2005, August 30). Cultural variation in eye movements during scene perception. PNAS, 102(35), 12629-12633.

Clay, V., König, P., König, S. (2019). Eye Tracking in Virtual Reality. Journal of Eye Movement Research, 12(1), 1-18.

Colter, A., Summers, K. (2014). Low Literacy Users. In Book: Eye Tracking in User Experience Design (Ed. by Bergstrom, J.C.R. and Schall, A. J.), Ch. 13, pp.331-350. Elsevier: Morgan Kaufman.

Constine, J. (2016). How Facebook News Feed Works. Available at: https://techcrunch.com/2016/09/06/ultimate-guide-to-the-newsfeed/.

Danielson, R. (1997). Learning styles, media preferences, and adaptive education. In: Proceedings of Workshop "Adaptive Systems and User Modeling on the World Wide Web", the 6th Int. Conf. on User Modeling, Chia Laguna, Sardinia, Italy, 31-35. 
Davies, A., Harper, S., Jay, C., Brown, A. (2016). Computational Methods for Analysis of Visual Behavior using Eye-tracking. In: Spink, A.J. (Ed.), Proceedings of Measuring Behavior, 2016, Dublin, Ireland, 1-4.

Djamasbi, S., Hall-Phillips, A. (2014). Visual Search. In Book: Eye Tracking in User Experience Design (Ed. by Bergstrom, J.C.R. and Schall, A.J.): Ch. 2, pp. 27-48. Elsevier: Morgan Kaufman.

Doak, C, Doak, L, Root, J. (1996). Teaching Patients with Low Literacy Skills, Second ed., Philadelphia: J.B. Lippincott Company.

Dorr, M., Vig, E., Gegenfurtner, K.R., Martinetz, T., Barth, E. (2008). Eye movement modelling and gaze guidance. The Fourth International Workshop on Human-Computer Conversation, Oxford, UK, 1-4.

Duchowski, A. (2017). Eye Tracking Methodology - Theory and Practice, Third ed. Springer.

EARLI SIG-27 (2017). EARLI Special Interest Group 27 - Online Measures of Learning Processes. Available at: https://www.earli.org/node/50

Engbert, R., Longtin, A., Kliegl, R. (2002). A dynamical model of saccade generation in reading based on spatially distributed lexical processing. Vision Research, 42, 621-636.

Eraslan, S., Yesilada, Y., Harper, S. (2016). Scanpath Trend Analysis on Web Pages: Clustering Eye Tracking Scanpaths. In: ACM Transactions on the Web, 10(4), 1-36.

Faraday, P. (2000). Visually critiquing web pages. In: Proceedings of the Eurographics Workshop, Milano, Italy, September 7-8, 1999. Springer, Vienna, 155-166.

Farnsworth, B. (2018). Eye Tracking - The Complete Pocket Guide. Blog at iMotions. Available at: https://imotions.com/blog/eye-tracking/

Fernandes, T. (1995), Global Interface Design: A Guide to Designing International User Interfaces, AP Professional, Chestnut Hill, MA.

Gilbert, J.E. and Han, C.Y. (1999). Arthur: adapting instruction to accommodate learning style. In: Proceedings of World Conference of the WWW and Internet (WebNet'99), Oct. 24-30, 1999, Honolulu, HI, AACE, 433-438.

Glenstrup, A.J. (1995). Eye Controlled Media: Present and Future State. PhD thesis, Laboratory of Psychology, University of Copenhagen.

Graf, S., Liu, T., Kinshuk, C. (2010). Analysis of learners' navigational behaviour and their learning styles in an online course. Journal of Computer Assisted Learning, 26(2), 116-131.

Guan, Y.-H., Hsu, H.-Y. (2015). Do Chinese readers rely on phonological recoding when comprehending English texts? In: Abstracts of the 18th European Conference on Eye Movements (ETRA-2015), August 16-21, 2015, Vienna, Austria, p.23.

Gudinavičius, A., Šuminas, A. (2018). Choosing a book by its cover: analysis of a reader's choice. Journal of Documentation, 74(2), 430-446. Available at: https://doi.org/10.1108/JD-09-2016-0111.

Hansen D.W, Majaranta, P. (2012). Basics of camera-based gaze tracking. In: Majaranta, P. et al. (Ed.) Gaze interaction and applications of eye tracking: advances in assistive technologies. Medical Information Science Reference, Hershey, 21-26.

Hansen D.W., Pece A.E.C. (2005). Eye tracking in the wild. Comput Vis Image Underst, 98(1), $155-181$.

Heimgärtner, R. (2007). Towards Cultural Adaptability in Driver Information and Assistance Systems. In: Aykin, N. (ed.) Usability and Internationalization, Part II, HCII 2007, LNCS 4560, 372-381, Springer, Heidelberg.

Heimgartner, R. (2013) Reflections on a Model of Culturally Influenced Human Computer Interaction to Cover Cultural Contexts in HCI Design. Int. Journal of Human-Computer Interaction, 29, 205-219.

Heimgärtner, R. (2014). ISO 9241-210 and Culture? - The Impact of Culture on the Standard Usability Engineering Process. In: A. Marcus (Ed.): DUXU 2014, Part IV, LNCS 8520, 3948, Springer International Publishing Switzerland.

Heimgärtner, R., Solanki, A. (2014). Using Agile Methods in Intercultural HCI Design Projects. In: A. Marcus (Ed.): DUXU 2014, Part I, LNCS 8517, 123-129, Springer International Publishing Switzerland. 
Heimgärtner, R., Sturm. C., Womser-Hacker, C., Linxen, S., Mandl, T. (2018). Interkulturelle Erforschung und Gestaltung von Benutzungsschnittstellen (UI) und Benutzererfahrung (UX) (German). In: die Gesellschaft für Informatik R. Dachselt, G. Weber (Ed.): Mensch und Computer 2018 - Workshopband, 02-05 September 2018, Dresden, 1-6.

Hofstede, G., Hofstede, G.J. (2010). Cultures and Organizations: Software of the Mind. McGrawHill, New York.

Holmqvist, K., Nyström, M., Andersson, R., Dewhurst, R., Jarodzka, H., Van de Weijer, J. (2011). Eye tracking: A comprehensive guide to methods and measures. Oxford: Oxford University Press.

Holmqvist, K., Nystrom, M., Mulvey, F. (2012). Eye tracker data quality: What it is and how to measure it. In: Proceedings of the symposium on eye tracking research and applications, 45 52.

Honold, P. (1999). Cross-cultural Usability Engineering: Development and State of the art. In: Proceedings of the 8th Int. Conf. on Human-Computer Interaction: Ergonomics and User Interfaces, L. Erlbaum Associates Inc., I, 1232-1236.

Hyönä, J. (2010). The use of eye movements in the study of multimedia learning. Learning and Instruction, 20(2), 172-176.

iMotions (2018). Eye tracking: Complete pocket guide. Available at: https://imotions.com/guides/eye-tracking/.

iMotions (2019). Combine biosensors and uncover underlying emotions and responses. Available at: https://imotions.com/biosensors/.

Jacob, R., Karn, K.S. (2003). Eye tracking in human-computer interaction and usability research: Ready to deliver the promises. In: Hyönä, J., Radach, R. \& Deubel, H. (Eds.), The mind's eye: Cognitive and applied aspects of eye movement research, 573-605. Oxford: Elsevier.

Jarrett C. (2012). Buttons on forms and surveys: a look at some research. In: Presentation at the Information Design Association conference, Greenwich, UK. Available at: http://www.slideshare. net/cjforms/buttons-on-forms-andsurveys-a-look-at-some-research-2012

Jarodzka, H., Balslev, T., Holmqvist, K., Nyström, M., Scheiter, K., Gerjets, P., Eika, B. (2012). Conveying clinical reasoning based on visual observation via eye-movement modelling examples. Instructional Science, 40(5), 813-827.

Jarodzka, H., Holmqvist, K., Gruber, H. (2017). Eye tracking in Educational Science: Theoretical frameworks and research agendas. Journal of Eye Movement Research, 10(1):3, 1-18.

Jarodzka, H., Van Gog, T., Dorr, M., Scheiter, K., Gerjets, P. (2013). Learning to see: Guiding students' attention via a model's eye movements fosters learning. Learning and Instruction, 25, 62-70.

Jbara, A., Feitelson, D.G. (2015). How programmers read regular code: a controlled experiment using eye tracking. In: Proceedings of the IEEE 23rd International Conference on Program Comprehension (ICPC '15). IEEE Press, Piscataway, NJ, USA, 244-254.

Johnstone CJ-M. (2006). Using the Think Aloud Method (Cognitive Labs) to Evaulate Test Design for Students with Disabilities and English Language Learners. Minneapolis, MN: University of Minnesota, National Center on Educational Outcomes; University of Minnesota.

Jucks, R., Schulte-Löbbert, P., Bromme, R. (2007). Supporting experts' written knowledge communication through reflective prompts on the use of specialist concepts. Journal of Psychology, 215(4), 237-247.

Just, M., Carpenter, P. (1980). A theory of reading: From eye fixations to comprehension. Psychological Review, 87(4), 329-354.

Kaššák, O., Kompan, M, Bielikova, M. (2016). Student Behavior in a Web-Based Educational System: Exit Intent Prediction. Int. Sc. Journal Engineering Applications of Artificial Intelligence, Elsevier; Issue: Mining the Humanities: Technologies and Applications, 51, $136-149$. 
Kelly, D. J., Miellet, S., Caldara, R. (2010, April 29). Culture shapes eye movements for visually homogeneous objects. Frontiers in Psychology, 1(6), 1-7.

Kirsch, I. (2002). Adult literacy in America: A First Look at the Results of the National Adult Literacy Survey. National Center for Education Statistics Washington, DC: U.S. Department of Education.

Krosnick, J.A. (1991). Response strategies for coping with the cognitive demands of attitude measures in surveys. Appl Cognit Psychol. 5(3), 213-236.

Lai, M.-L., Tsai, M.-J., Yang, F.-Y., Hsu, C.-Y., Liu, T.-C., Lee, S. W.-Y., Lee, M.-H., Chiou, G.L., Liang, J.-C., Tsai, C.-C. (2013). A review of using eye-tracking technology in exploring learning from 2000 to 2012. Educational Research Review, 10, 90-115.

Leff, D.R., James, D.R., Orihuela-Espina, F., Kwok, K.-W., Sun, L.W., Mylonas, G., Thanos, A., Ara, W.D., Yang, G.-Z. (2015). The impact of expert visual guidance on trainee visual search strategy, visual attention and motor skills. Frontiers in Human Neuroscience, 9(526), $1-11$.

Lim, H., Fussell, S.R. (2017). Understanding How People Attend to and Engage with Foreign Language Posts in Multilingual Newsfeeds. In: Proceedings of the 11th International AAAI (Association for the Advancement of Artificial Intelligence) Conference on Web and Social Media (ICWSM 2017), 588-591.

Litchfield, D., Ball, L.J. (2011). Using another's gaze as an explicit aid to insight problem solving. The Quarterly Journal of Experimental Psychology, 64(3), 649-656.

Liu, H.-C., Lai, M.-L., Chuang, H.-H. (2011). Using eye-tracking technology to investigate the redundant effect of multimedia web pages on viewers' cognitive processes. Computers in Human Behavior, 27(6), 2410-2417.

Mancheva, L., Lemaire, B., Guerin-Dugue, A., Ecalle, J., Valdois, S. (2015). Individual differences in language skills affect children's eye movement characteristics. In: Abstracts of the 18th European Conference on Eye Movements (ETRA-2015), August 16-21, 2015, Vienna, Austria, p. 24.

Mason, L., Tornatora, M. C., Pluchino, P. (2013). Do fourth graders integrate text and picture in processing and learning from an illustrated science text? Evidence from eye-movement patterns. Computers \& Education, 60(1), 95-109.

Mayer, R. (2009). Multimedia Learning. Second ed. Cambridge, UK: Cambridge University Press.

McIntyre, N.A., Jarodzka, H., Klassen, R.M. (2019). Capturing teacher priorities: Using real-world eye-tracking to investigate expert teacher priorities across two cultures. Learning \& Instruction; 60, 215-224.

McNamara, A., Booth, T., Sridharan, S., Caffey, S., Grimm, C., Bailey, R. (2012). Directing gaze in narrative art. In: Proceedings of the ACM Symposium on Applied Perception (SAP '12). Association for Computing Machinery, New York, NY, USA, 63-70.

McNamara, L.A., Stracuzzi, D., Czuchlewski, K.R. (2015). Challenges in Eyetracking Data Analysis: From the Laboratory to the Wild World of Information. In: Abstracts of the 18th European Conference on Eye Movements (ETRA-2015), August 16-21, 2015, Vienna, Austria, p.26.

Meisner, M., and Oll, J. (2019). The Promise of Eye-Tracking Methodology in Organizational Research: A Taxonomy, Review, and Future Avenues. Organizational Research Methods, 22(2), 590-617.

Miyahira, A., Morita, K., Yamaguchi, H., Nonaka, K., Maeda, H. (2000). Gender differences of exploratory eye movements. Life Sciences, 68(5), 569-577.

Mohamed, A., Da Silva, M.P., Courboulay, V. (2008). A history of eye gaze tracking. Technical Report; hal-00215967, version 1 - 24 Jan 2008.

Mueller, S., Jackson, C., Skelton, R. (2008). Sex differences in a virtual water maze: An eye tracking and pupillometry study. Behavioural Brain Research, 193, 209-215.

Nagle, M, Zietlow, E. (2012). An Exploratory Comparison of Two Website Usability Testing Methods: Concurrent Think-Aloud and Eye-Tracking with Retrospective Think-Aloud. University of Baltimore. 
Nakayama, M., Shimizu, Y. (2006). Evaluation of a Multimedia Learning Exercise using Oculomotors. In: ACM Eye Tracking Reseach \& Applications Symposium 2006, ACM Press (434061), p.46.

Nielsen, J. (2006). F-Shaped Pattern for Reading Web Content (original study). Available at: https://www.nngroup.com/articles/f-shaped-pattern-reading-webcontent-discovered/.

Nielsen, J. (2017). F-Shaped Pattern of Reading on the Web: Misunderstood, But Still Relevant (Even on Mobile). Available at: https://www.nngroup.com/articles/fshaped-pattern-reading-web-content/.

Nyström, M., Holmqvist, K. (2008). Semantic override of low-level features in image viewing both initially and overall. Journal of Eye Movement Research, 2, 1-11.

Oliva, M., Niehorster, D.C., Jarodzka, H., Holmqvist, K. (2017). Influence of coactors on saccadic and manual responses. $i$-Perception, 8(1), 1-23.

Partala, T., Jokiniemi, M., Surakka, V. (2000, November 6-8). Pupillary Responses to Emotionally Provocative Stimuli. In: Proc. of Eye Tracking Research and Applications Symposium (ETRA 2000), 123-129.

Plocher, T., Rau, P-L, P., Choong, Y-Y. (2012). Cross-Cultural Design. In: Salvendy (ed.): Handbook of Human Factors and Ergonomics, Fourth Edition, Ch.6, pp. 162-191. John Wiley \& Sons, Inc.

Rakoczi, G. (2010). Cast your Eyes on Moodle: An Eye Tracking Study investigating learning with Moodle. The 4th Int. Conf. Proceedings - Moodle.si2010, Koper, 21 May 2010, 203213.

Raschke, M., Blascheck, M., Burch, M. (2014). Visual Analysis of Eye Tracking Data. Springer Science + Business Media New York, In: W. Huang (ed.), Handbook of Human Centric Visualization, Ch.15, 391-409.

Rayner, K. (1998). Eye movements in reading and information processing: 20 years of research. Psychological Bulletin, 124(3), 372-422.

Rayner, K. (2009). Eye movements and attention in reading, scene perception, and visual search. The Quarterly Journal of Experimental Psychology, 62(8), 1457-1506.

Rayner, K., Chace, K.H., Slattery, T.J., Ashby, J. (2006). Eye movements as reflections of comprehension process in reading. Scientific Studies of Reading, 10(3), 241-255.

Rayner, K., Pollatsek, A., Ashby, J., Clifton Jr., C. (2012). Psychology of Reading: 2nd Edition. Ch.10, Stages of Reading Development, 279-308. New York, NJ: Psychology Press.

Rayner, K., Sereno, S.C., Raney, G.E. (1996). Eye movement control in reading: A comparison of two types of models. Journal of Experimental Psychology: Human Perception and Performance, 22, 1188-1200.

Reichle, E.D., Pollatsek, A., Fisher, D.L., Rayner, K. (1998). Toward a model of eye movement control in reading. Psychological Review, 105, 125-157.

Reichle, E.D., Rayner, K., Pollatsek, A. (2003). The EZ-Reader model of eye movement control in reading: Comparisons to other models. Behavioral and Brain Sciences, 26, 445-476.

Romano, J., Chen, J. (2011). A Usability and Eye-Tracking Evaluation of Four Versions of the Online National Survey of College Graduates (NSCG): Iteration 2. In: Report - Study Series (Survey Methodology \#2011-01), Center for Survey Measurement, Research and Methodology Directorate, U.S. Census Bureau Washington, D.C., 1-49. Available at: http://docplayer.net/1219379-Study-series-survey-methodology2011-01.html

Rosch, J.L., Vogel-Walcutt, J.J. (2013). A review of eye-tracking applications as tools for training. Cognition, Technology and Work, 15(3), 313-327.

Röse, K. (2005). The Development of Culture-Oriented Human Machine Systems: Specification, Analysis and Integration of Relevant Intercultural Variables. In: Kaplan, M. (ed.) Cultural Ergonomics, Advances in Human Performance and Cognitive Engineering Research. Amsterdam: Elsevier. 
Schoper, Y., Heimgärtner, R. (2013). Lessons from Intercultural Project Management for the Intercultural HCI Design Process. In: Marcus, A. (ed.) DUXU/HCII 2013, Part II. LNCS, 8013, 95-104. Springer, Heidelberg.

Scott, D., Findlay J.M. (1993). Visual search, eye movements and display units, human factors report. Technical report, University of Durham.

Sharafi, Z., Soh, Z., Guéhéneuc, Y. (2015). A systematic literature review on the usage of eyetracking in software engineering. Information and Software Technology, 67, 79-107.

She, H.-C., Chen, Y.-Z. (2009). The impact of multimedia effect on science learning: Evidence from eye movements. Computers \& Education, 53(4), 1297-1307.

Slykhuis, D., Wiebe, E., Annetta, L. (2005). Eye-tracking students' attention to PowerPoint photographs in a science education setting. Journal of Science Education and Technology, 14(5/6), 509-520.

Skuballa, I. T., Fortunski, C., Renkl, A. (2015). An eye movement pre-training fosters the comprehension of processes and functions in technical systems. Frontiers in Psychology, 6 (598), 1-13.

Skuballa, I. T., Schwonke, R., Renkl, A. (2012). Learning from narrated animations with different support procedures: Working memory capacity matters. Applied Cognitive Psychology, 26(6), 840-847.

Sridharan, S., Bailey, R., McNamara, A., Grimm, C. (2011). Subtle gaze manipulation for improved mammography training. In: Proceedings of the ACM SIGGRAPH Symposium on Applied Perception in Graphics and Visualization, APGV '11, August 2011, pp. 112.

Summers, K., Langford, J, Wu, J, Abela, C, Souza, R. (2006). Designing web-based forms for users with lower literacy skills. Proc Am Soc Info Sci Tech, 43(1), 1-12.

Summers, K., Summers, M. (2004). Making the web friendlier for lower-literacy users. Intercom; 51(6), 19-21.

Summers, K., Summers, M. (2005). Reading and navigational strategies of web users with lower literacy skills. Proc Am Soc Info Sci Tech, 42(1), 1-11.

Thomas, A., Kinast, E.-U., Schroll-Machl, S. (2010). Handbook of intercultural communication and cooperation. Basics and Areas of Application. Vandenhoeck \& Ruprecht, Göttingen.

Tobii Technology (2010). An Introduction to Eye Tracking and Tobii Eye Trackers. Whitepaper. Available at: http://www.123seminarsonly.com/Seminar-Reports/201311/25907389-Tobii-Eye-Tracking.pdf

Toker, D., Conati, C., Steichen, B., Carenini, G. (2013). Individual user characteristics and information visualization: Connecting the dots through eye tracking. In: Proceedings of the SIGCHI Conference on Human Factors in Computing Systems CHI '13, 295-304.

Tsianos, N., Germanakos, P., Lekkas, Z., Mourlas, C., Samaras, G.S. (2009). Eye-tracking users' behavior in relation to Cognitive Style within an e-Learning environment. In: Proceedings of the 9th IEEE Int. Conf. on Advanced Learning Technologies (ICALT 2009), 329-333.

Turenko, V., Baltulionis, S., Vasiljevas, M., Damaševičius, R. (2019). Analysing program source code reading skills with eye tracking technology. In: Proceedings of the IVUS International Conference on Information Technology (IVUS 2019), Kaunas, Lithuania, April 25, 2019. 33-37.

Van Gog, T., Jarodzka, H., Scheiter, K., Gerjets, P., Paas, F. (2009). Attention guidance during example study via the model's eye movements. Computers in Human Behavior, 25(3), 785791.

Vöhringer-Kuhnt, T. (2002). The Influence of Culture on Usability. Freie Universität Berlin.

Vorstius, C., Kim, Y.-S., Radach, R. (2015). Eye movement control in oral vs. silent reading: Evidence from a large sample of very young readers. In: Abstracts of the 18th European Conference on Eye Movements (ETRA-2015), August 16-21, 2015, Vienna, Austria, p.23.

White, S.A. (2003). Framework for the 2003 National Assessment of Adult Literacy (NCES 2005531). Washington, DC: U.S. Department of Education.

White, S.J., Liversedge, S.P. (2006). Linguistic and nonlinguistic influences on the eyes' landing positions during reading. The Quarterly Journal of Experimental Psychology, 59(4), 760782. 
White, S.J., Liversedge, S.P. (2004). Orthographic familiarity influences initial eye fixation positions in reading. European Journal of Cognitive Psychology, 16, 52-78.

Yang, F.Y., Chang, C. Y., Chien, W.R., Chien, Y.T., Tseng, Y.H. (2013). Tracking learners' visual attention during a multimedia presentation in a real classroom. Computers \& Education, 62, 208-220.

Yang, F-Y, Huang, R-T, Tsai, I-J. (2016). The Effects of Epistemic Beliefs in Science and Gender Difference on University Students' Science-Text Reading: An Eye-Tracking Study. Int. Journal of Science and Mathematics Education, 14, 473-498.

Yenigalla L., Sinha V., Sharif B., Crosby M. (2016). How Novices Read Source Code in Introductory Courses on Programming: An Eye-Tracking Experiment. In: Schmorrow D., Fidopiastis C. (eds) Foundations of Augmented Cognition: Neuroergonomics and Operational Neuroscience. AC 2016. LNCS 9744, 120-131. Springer, Cham. https://doi.org/10.1007/978-3-319-39952-2_13.

Zuga, B., Kapenieks, A., Gorbunovs, A., Jirgensons, M., Kapenieks, J., Kapenieks, J., Vitolina, I., Jakobsone-Snepste, G., Kudina, I., Kapenieks, K., Timsans, Z., Gulbis, R. (2015). Concept of Learner Behaviour Data Based Learning Support. In Book series: Procedia Computer Science, 43, 134-140.

Received August 3, 2020, revised November 19, 2020, accepted January 10, 2021 